\title{
Adaptive Disturbance Tracking Theory with State Estimation and State Feedback for Region II Control of Large Wind Turbines
}

\author{
Mark J. Balas, Kaman S. Thapa Magar, and Susan A. Frost
}

\begin{abstract}
A theory called Adaptive Disturbance Tracking Control (ADTC) is introduced and used to track the Tip Speed Ratio (TSR) of 5 MW Horizontal Axis Wind Turbine (HAWT). Since ADTC theory requires wind speed information, a wind disturbance generator model is combined with lower order plant model to estimate the wind speed as well as partial states of the wind turbine. In this paper, we present a proof of stability and convergence of ADTC theory with lower order estimator and show that the state feedback can be adaptive.
\end{abstract}

\section{INTRODUCTION}

Large wind turbines are operated in three different regions called Region I, Region II and Region III, and the available wind speed determines the region of operation. Region I is the startup region and wind speed is not sufficient to produce the power. When wind speed becomes large enough to produce the power but not enough to produce the rated power, then it operates in Region II. In Region III, wind speed is normally larger than the rated wind speed and produces the rated power.

The amount of power that can be captured using wind turbine is given by [1]:

$$
P=\frac{1}{2} \rho A C_{P} \omega^{3}
$$

Where, $\mathrm{P}$ is the power, $\mathrm{A}$ is the area of the rotor disc, $\mathrm{C}_{\mathrm{P}}$ is the power coefficient, $\rho$ is the air density, $\omega$ and is the freestream wind velocity.

The power coefficient $C_{P}$ is the function of tip-speed ratio $(\lambda)$ and the blade pitch angle $(\beta)$. The Tip Speed Ratio (TSR) can be expressed as:

$$
\lambda \equiv \frac{\Omega R}{\omega}
$$

*Research supported by Wind Energy Research Center, University of Wyoming.

Mark J. Balas is with Department of Electrical Engineering, University of Wyoming, Laramie, WY 82070 USA (phone: 307-766-5599; e-mail: mbalas@uwyo.edu).

Kaman S. Thapa Magar is with the Department of Electrical Engineering, University of Wyoming, Laramie, WY 82070 USA (e-mail: kthapama@uwyo.edu).

Susan A. Frost is with the NASA Ames Research Center, Moffett Field, CA, 95034 (Susan.A.Frost@nasa.gov).

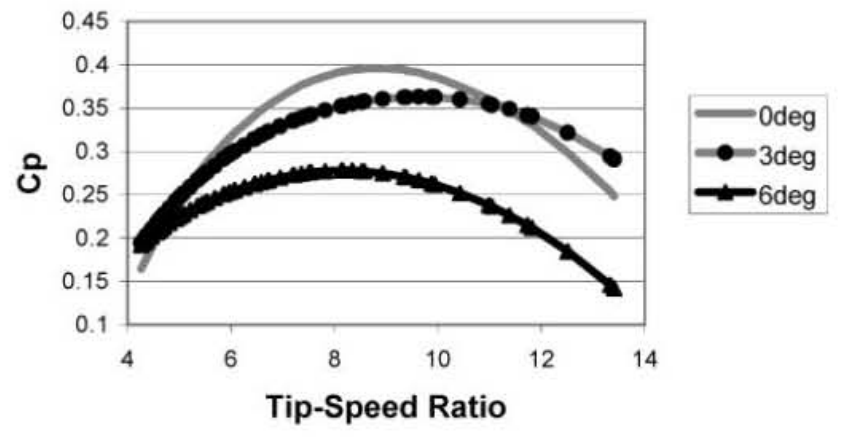

Figure 1 Plot of $C_{P}$ vs Tip - Speed Ratio for different Blade Pitch Angle

Where, $\Omega$ is the rotor speed, $\mathrm{R}$ is the rotor radius.

The variation of power coefficient $\left(C_{p}\right)$ with TSR for fixed sets of blade pitch angle is in figure 1[2]. From the figure, it can be seen that, for a certain blade pitch angle, there exist an optimum TSR for which $\mathrm{C}_{\mathrm{p}}$ is maximum. In Region II, the wind speed is not sufficient to produce the rated power so the TSR must be maintained constant at its optimum value such that the power coefficient is maximum, which ultimately maximizes the power captured by wind turbine.

From equation (2), the TSR is a function of the rotor speed and the wind speed. Since variation in the wind speed have effect on rotor speed, the rotor speed must be varied in same proportion to keep the TSR constant.

The foundation of counteracting the persistence disturbance in a plant was developed by Johnson [3]. Balas refined this idea [4], and developed a new idea of Disturbance Tracking Control and used for Region II control of the wind turbine. The motivation behind the theory of Disturbance Tracking Control is to make the wind turbine track the wind speed, which ultimately tries to keep the TSR constant at some optimum value.

The TSR tracking error $(\varepsilon)$ is introduced as the deviation of the actual TSR $(\lambda)$ from the operating or optimum TSR $\left(\lambda_{\mathrm{OP}}\right)$.

$$
\varepsilon \equiv \lambda-\lambda_{o p}=\frac{\Omega R}{\omega}-\frac{\Omega_{o p} R}{\omega_{o p}}=\frac{R}{\omega}\left(\Omega-\frac{\Omega_{o p}}{\omega_{o p}} \omega\right)
$$


where $\left(\Omega_{\mathrm{op}}, \omega_{\mathrm{op}}\right)$ is the desired turbine operating point corresponding to the desired TSR $\left(\lambda_{\mathrm{OP}}\right)$. We let the Output Tracking Error be

$$
\left\{\begin{array}{l}
e_{y} \equiv \Omega_{T}-Q^{*} \omega \\
\text { with } Q \equiv \frac{\Omega_{o p}}{\omega_{o p}}
\end{array}\right.
$$

where $(\mathrm{Q})$ is the tracking ratio.

and think of $(\Omega)$, the turbine speed, as a measured output of the turbine and $(\omega)$, the wind speed, as a disturbance on the turbine. Then DTC uses a feedback control law that produces:

$$
e_{y} \equiv \Omega-Q^{*} \omega \underset{t \rightarrow \infty}{\longrightarrow} 0
$$

This (approximately) produces tracking of the desired TSR: $\varepsilon \equiv \lambda-\lambda_{o p} \underset{t \rightarrow \infty}{\longrightarrow} 0[5]$.

\section{Adaptive Disturbance Tracking Control With Partial State Estimation and State FeEdback}

In this section we introduce further modification of the theory presented in [5] with addition of wind speed and partial states estimation, and partial state feedback.

The wind turbine is assumed to be modeled by a linear, time-invariant, finite-dimensional system:

$$
\left\{\begin{array}{l}
\dot{x}=A x+B u_{p}+\Gamma u_{D} \\
y=C x ; x(0)=x_{0}
\end{array}\right.
$$

and the plant model with partial states of (6a) is expressed as:

$$
\left\{\begin{array}{l}
\dot{x}_{m}=A_{m} x_{m}+B_{m} u_{p}+\Gamma_{m} u_{D} \\
y_{m}=C_{m} x_{m} ; x_{m}(0)=x_{0}
\end{array}\right.
$$

where, the plant state, $\mathrm{x}$, is an $\mathrm{N}_{\mathrm{p}}$-dimensional vector, the control input vector, $\mathrm{u}_{\mathrm{p}}$, is $\mathrm{M}$-dimensional, the sensor output vector, $\mathrm{y}$, is $\mathrm{P}$-dimensional. $\mathrm{x}_{\mathrm{m}}$ is $\mathrm{m}$-dimensional $(\mathrm{m}<\mathrm{n})$ lower order plant model state vector, $y_{m}$ is the $P_{m}$ dimensional model output vector. A, B, C, $\Gamma$ are the state, input, output and disturbance matrix of plant with appropriate dimensions. $A_{m}, B_{m}, C_{m}, \Gamma_{m}$ are the state, input, output and disturbance matrix of the lower order plant model with appropriate dimensions. The disturbance input vector, $\mathrm{u}_{\mathrm{D}}$, is $\mathrm{M}_{\mathrm{D}}$-dimensional and will be thought to come from the Disturbance Generator:

$$
\left\{\begin{array}{l}
u_{D}=\theta z_{D} \\
\dot{z}_{D}=F z_{D} ; z_{D}(0)=z_{0}
\end{array}\right.
$$

where, the disturbance state, $\mathrm{Z}_{\mathrm{D}}$, is $\mathrm{N}_{\mathrm{D}}$-dimensional.
All matrices in Eqs. (6)-(7) have the appropriate compatible dimensions. Such descriptions of persistent disturbances were first used in [5] to describe signals of known form but unknown amplitude. Equation (7) can be rewritten in a form that is not a dynamical system, which is sometimes easier to use:

$$
\left\{\begin{array}{l}
u_{D}=\Theta z_{D} \\
z_{D}=L \varphi_{D}
\end{array}\right.
$$

where, $\varphi_{\mathrm{D}}$ is a vector composed of the known basis functions for the solution of $u_{D}=\theta z_{D}$, i.e., $\varphi_{D}$ are the basis functions which make up the known form of the disturbance, and $\mathrm{L}$ is a matrix of appropriate dimension. The method for tracking persistent disturbances used in this paper requires only the knowledge of the form of the disturbance, the amplitude of the disturbance does not need to be known, i.e. $(\mathrm{L}, \theta)$ are unknown. In this paper, we will be interested in rejecting step disturbances of unknown amplitude which can be represented in the form of Eq. (8) as $\varphi_{D}=1$, with $(L, \theta)$ unknown. This has been a viable model for wind fluctuations in our previous work.

Now combining equation (6b) and (7) we get a new augmented plant model [6]:

$$
\left[\begin{array}{c}
\dot{x}_{m} \\
\dot{z}_{D}
\end{array}\right]=\left[\begin{array}{cc}
A_{m} & \Gamma_{m} \theta \\
F & 0
\end{array}\right]\left[\begin{array}{c}
x_{m} \\
z_{D}
\end{array}\right]+\left[\begin{array}{c}
B_{m} \\
0
\end{array}\right] u
$$

In equation (9) we used the lower order plant model to estimate the partial state and use the partial state feedback.

Using the augmented plant model in (9), a state estimator can be designed as:

$\left[\begin{array}{c}\dot{\hat{x}}_{m} \\ \dot{\hat{z}}_{D}\end{array}\right]=\left[\begin{array}{cc}A_{m} & \Gamma_{m} \theta \\ F & 0\end{array}\right]\left[\begin{array}{l}\hat{x}_{m} \\ \hat{z}_{D}\end{array}\right]+\left[\begin{array}{c}B_{m} \\ 0\end{array}\right] u+\left[\begin{array}{l}K_{x} \\ K_{D}\end{array}\right]\left(y_{p}-\hat{y}_{m}\right)$

The estimator equation (10) can also be broken down into wind disturbance estimator and state estimator as:

$\dot{\hat{x}}_{m}=A_{m} \hat{x}_{m}+B_{m} u+\Gamma_{m} \theta \hat{z}_{D}+K_{x}\left(y_{p}-\hat{y}_{m}\right)$

$\dot{\hat{z}}_{D}=F \hat{z}_{D}+K_{D}\left(y_{p}-\hat{y}_{m}\right)$

Using the wind disturbance state estimation, the estimated wind speed can be expressed as:

$$
u_{D}=\theta \hat{z}_{D}
$$

Our control objective will be to cause the output of the plant, $y_{p}$, to asymptotically track some linear function of 
estimated disturbances of the form given by the disturbance estimator. We define the estimated output tracking error vector as:

$$
\hat{e}_{y} \equiv y_{p}-Q u_{D}
$$

To achieve the desired control objective, we want $\hat{e}_{y} \underset{t \rightarrow \infty}{\longrightarrow} 0$

This aligns with the TSR tracking in Region II described by equation (5).

Consider the plant given by Eq. (6a) with the disturbance generator given by Eq. (7) and respective disturbance and state estimator given by (10) and (11). Our control objective for this system will be accomplished by an Adaptive Control Law of the form:

$u_{P}=G_{e} \hat{e}_{y}+G_{D} \varphi_{D}+G_{x} x_{m}$

where $G_{e}$ and $G_{D}$ are adaptive gain matrices of the appropriate compatible dimensions, and $G_{x}$ is the state feedback gain matrix.

Now we specify the Adaptive Gain Laws, which will produce asymptotic tracking:

$$
\left\{\begin{array}{l}
\dot{G}_{e}=-\hat{e}_{y} \hat{e}_{y}^{T} \gamma_{e} \\
\dot{G}_{D}=-\hat{e}_{y} \varphi_{D}^{T} \gamma_{D}
\end{array}\right.
$$

where $\gamma_{e}, \gamma_{D}$ are arbitrary, positive definite matrices. Our Adaptive Controller is specified by Eq. (14) with the above adaptive gain laws Eq. (15).

\section{Stability and CONVERGENCE ANaLysis}

\section{A. Problem Formulation}

Assume a linear, time invariant and finite dimensional plant of (6a) and disturbance generator of (7)

We define the estimator as:

$$
\left\{\begin{array}{c}
\dot{\hat{z}}=L_{21} y+L_{22} \hat{z}+L_{23} u \\
\text { where } \hat{z} \equiv T w+e \\
z
\end{array}\right.
$$

Where,

$$
w \equiv\left[\begin{array}{c}
x \\
z_{D}
\end{array}\right]
$$

$$
\Rightarrow\left\{\begin{array}{l}
\dot{w}=\underbrace{\left[\begin{array}{cc}
A & \Gamma \theta \\
0 & F
\end{array}\right]}_{\bar{A}} w+\left[\begin{array}{c}
B \\
0
\end{array}\right] \\
y=\left[\begin{array}{cc}
C & 0
\end{array}\right] w \\
e_{y}=\underbrace{\left[\begin{array}{cc}
C & -Q \theta
\end{array}\right]}_{\bar{B}} w
\end{array}\right.
$$

Define the ideal trajectories as:

$$
\left\{\begin{array}{l}
\dot{w}_{*}=\bar{A} w_{*}+\bar{B} u_{*} \\
y_{*}=\left[\begin{array}{ll}
C & 0
\end{array}\right] w_{*} \\
e_{y}^{*}=\bar{C} w_{*}=0
\end{array}\right.
$$

with

$$
\begin{aligned}
& \left\{\begin{array}{l}
w_{*} \equiv\left[\begin{array}{l}
x_{*} \\
z_{D}
\end{array}\right]=\left[\begin{array}{l}
S_{1} \\
I_{D}
\end{array}\right] z_{D} \\
\overline{S_{1}} \\
u_{*} \equiv S_{2} z_{D}+G_{z}^{*} z_{*} ; z_{*} \equiv T w_{*}=T \overline{S_{1}} z_{D}
\end{array}\right. \\
& \Rightarrow u_{*} \equiv \underbrace{\left(S_{2}+G_{z}^{*} T \overline{S_{1}}\right)}_{S_{2}} z_{D}+G_{z}^{*} z_{*}
\end{aligned}
$$

let
$\left\{\begin{array}{l}\Delta w \equiv w-w_{*}=\left[\begin{array}{c}\Delta x \\ 0\end{array}\right] \\ \Delta u \equiv u-u_{*} \\ \Delta y \equiv y-y_{*} \\ \Delta e_{y} \equiv e_{y}-e_{y}^{*}=e_{y} \\ \Delta z \equiv z-z_{*}=T w-T w_{*}=T \Delta w\end{array}\right.$

$$
\Rightarrow\left\{\begin{array}{l}
\Delta \dot{w}=\bar{A} \Delta w+\bar{B} \Delta u \\
\Delta y=\left[\begin{array}{ll}
C & 0
\end{array}\right] \Delta w \\
e_{y}=\Delta e_{y}=\bar{C} \Delta w
\end{array}\right.
$$

now assume:

$z_{D}=H_{D} z$ or $H_{D} T=\left[\begin{array}{ll}0 & I_{D}\end{array}\right]$

$$
\begin{aligned}
& \Rightarrow \hat{z}_{D}=H_{D} \hat{z}=H_{D} T w+H_{D} e \\
& \therefore \hat{z}_{D}=z_{D}+H_{D} e
\end{aligned}
$$

define 


$$
\begin{gathered}
\left\{\begin{array}{l}
\hat{u}_{D} \equiv \theta \hat{z}_{D} \\
\hat{e}_{y} \equiv y-Q \hat{u}_{D}
\end{array}\right. \\
\hat{e}_{y} \equiv y-Q \theta \hat{z}_{D} \\
=\underbrace{y-Q \theta z_{D}}_{e_{y}}-Q \theta H_{D} e \\
=e_{y}-Q \theta H_{D} e \\
=\left(e_{y}-e_{y}^{*}\right)-Q \theta H_{D} e \\
=\Delta e_{y}-Q \theta H_{D} e \\
\Rightarrow \hat{e}_{y}=\bar{C} \Delta w-Q \theta H_{D} e
\end{gathered}
$$

$\Rightarrow \dot{e}=L_{22} e$

Let $T \equiv\left[\begin{array}{ll}T_{1} & T_{2}\end{array}\right]$ then $H_{D} T$

$$
=\left[\begin{array}{ll}
0 & I_{D}
\end{array}\right] \Rightarrow H_{D} T_{1}=0 \text { and } H_{D} T_{2}=I_{D} .
$$

Also,

$$
\begin{aligned}
L_{23} & =T \bar{B}=T_{1} B \text { and } T \ni T \bar{A}-L_{22} T \\
& =L_{21}\left[\begin{array}{ll}
C & 0
\end{array}\right] \Rightarrow\left\{\begin{array}{l}
T_{1} A-L_{22} T_{1}=L_{21} C \\
T_{2} F-L_{22} T_{2}+T_{1} \Gamma \theta=0
\end{array}\right.
\end{aligned}
$$

B. Closed Loop Stability Analysis:

We have,

adaptive Control law is:

$$
\begin{aligned}
& u=G_{e} \hat{e}_{y}+G_{D} \varphi_{D}+G_{z} \hat{z} \\
& \Delta u \equiv u-u_{*}=u-\bar{S}_{2} z_{D} \\
& =u-\left(S_{2} z_{D}+G_{z}^{*} z_{*}\right) \\
& =u-S_{2} L_{D} \varphi_{D}-G_{z}^{*} z_{*} \\
& =G_{e}^{*} \hat{e}_{\nu}+(\underbrace{G_{D}^{*}-S_{2} L_{D}}_{=0}) \varphi_{D}+G_{z}^{*} \hat{z}-G_{z}^{*} z \text {. }
\end{aligned}
$$

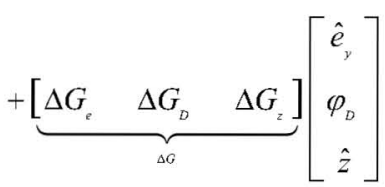

$$
\begin{aligned}
& =G_{e}^{*}\left(\bar{C} \Delta w-Q \theta H_{D} e\right)+G_{z}^{*}(T \Delta w+e)+\Delta G \eta \\
& =\left(G_{e}^{*} \bar{C}+G_{z}^{*} T\right) \Delta w+\left(G_{z}^{*}-G_{e}^{*} Q \theta H_{D}\right) e+h
\end{aligned}
$$

Where,

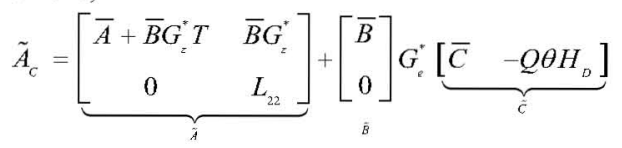

Lemma:

$(\tilde{A}, \tilde{B}, \tilde{C})$ is $\mathrm{ASPR} \Leftrightarrow(A, B, C)$ is ASPR

Proof:

$$
\begin{aligned}
& \tilde{C} \tilde{B}=\overline{C B}=C B \\
& \tilde{P}(s) \equiv \tilde{C}(s I-\tilde{A})^{-1} \tilde{B} \\
& =\underbrace{\left[\begin{array}{cc}
\bar{C} & -Q \theta H_{D}
\end{array}\right]}_{\tilde{C}}(s I-\underbrace{\left[\begin{array}{cc}
\bar{A}+\bar{B} G_{z}^{*} T & \bar{B} G_{z}^{*} \\
0 & L_{22}
\end{array}\right]}_{\tilde{A}})^{-1}\left[\begin{array}{c}
\bar{B} \\
0
\end{array}\right] \\
& =\bar{C}\left(s I-\left(\bar{A}+\bar{B} G_{z}^{*} T\right)\right)^{-1} \bar{B} \\
& =[\underbrace{\left[\begin{array}{cc}
C & -Q \theta
\end{array}\right]}_{C}\left(s I-\left[\begin{array}{cc}
A+B G_{z}^{*} T_{1} & \Gamma \theta+B G_{z}^{*} T_{2} \\
0 & F
\end{array}\right]\right)^{-1}\left[\begin{array}{l}
B \\
0
\end{array}\right]
\end{aligned}
$$

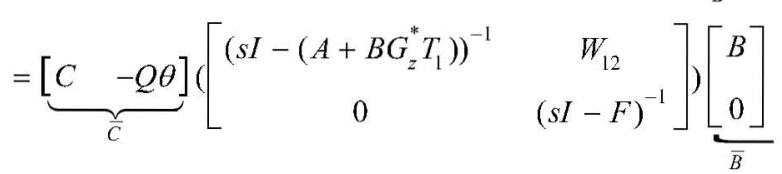

$$
\begin{aligned}
& =C\left(s I-\left(A+B G_{z}^{*} T_{1}\right)\right)^{-1} B \equiv P_{1}(s)
\end{aligned}
$$$$
\dot{e}=\dot{\hat{z}}-T \dot{w}
$$

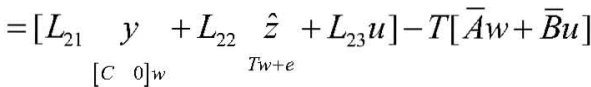$$
=\left(L_{21}\left[\begin{array}{ll}
C & 0
\end{array}\right]+L_{22} T-T \bar{A}\right) w+L_{22} e+\left(L_{23}-T \bar{B}\right) u
$$

Now use

$\left(A+B G_{z}^{*} T_{1}, B, C\right)$ is ASPR $\Leftrightarrow C B>0$

and $P_{1}(\mathrm{~s})$ minimum phase. 
But State Feedback does not change minimum phase of a transfer function, proof of this will be presented later; so $P(s)$ minimum phase $\Leftrightarrow P_{1}(s)$ minimum phase.

So $C B>0$ and $P(s)$ minimum phase will guarantee that $\exists G_{e}^{*} \ni\left(\tilde{A}+\tilde{B} G_{e}^{*} \tilde{C}, \tilde{B}, \tilde{C}\right)$ is SPR. Consequently, we have the following :

Theorem

1) $C B>0$ and $P(s)$ minimum phase

2) $\varphi_{\mathrm{D}}$ bounded

$\Rightarrow$ Adaptive Control

$\left\{\begin{array}{l}u=G_{e} \hat{e}_{y}+G_{D} \varphi_{D}+G_{z} \hat{z} \\ \dot{\hat{z}}=L_{21} y+L_{22} \hat{z}+L_{23} u\end{array}\right.$

with gain adaption laws

$\left\{\begin{array}{l}\dot{G}_{e}=-\hat{e}_{y} \hat{e}_{y}^{T} \gamma_{e} ; \gamma_{e}>0 \\ \dot{G}_{D}=-\hat{e}_{y} \varphi_{D}^{T} \gamma_{D} ; \gamma_{D}>0 \\ \dot{G}_{z}=-\hat{e}_{y} \hat{z}^{T} \gamma_{z} ; \gamma_{z}>0\end{array}\right.$

produces

$\left\{\begin{array}{l}\Delta w=\left[\begin{array}{c}\Delta x \\ 0\end{array}\right] \underset{t \leftarrow \infty}{\longrightarrow} 0 \\ e \stackrel{\longrightarrow \leftarrow \infty}{\longrightarrow} 0 \\ \hat{e}_{y}=\bar{C} \Delta w-Q \theta H_{D} e \underset{t \leftarrow \infty}{\longrightarrow} 0 \\ e_{y}=\bar{C} \Delta w \underset{t \leftarrow \infty}{\longrightarrow} 0\end{array}\right.$

with bounded adaptive gains

This does allow adaptive $G_{z}$.

C. Solvability of the Matching Conditions Ideal Trajectories:

$\left\{\begin{array}{l}\dot{w}_{*}=\bar{A} w_{*}+\bar{B} u_{*} \\ e_{y}^{*}=\bar{C} w_{*}=0\end{array}\right.$

with

$\left\{\begin{array}{l}w_{*} \equiv\left[\begin{array}{l}x_{*} \\ z_{D}\end{array}\right]=\left[\begin{array}{l}S_{1} \\ I_{D}\end{array}\right] z_{D} \\ u_{\bar{S}_{*}} \equiv S_{2} z_{D}+G_{z}^{*} T \bar{S}_{1} z_{D}=\underbrace{\left(S_{2}+G_{z}^{*} T \bar{S}_{1}\right)}_{\bar{S}_{2}} z_{D}\end{array}\right.$

$\Leftrightarrow\left\{\begin{array}{l}\bar{S}_{1} F=\bar{A} \bar{S}_{1}+\bar{B} \bar{S}_{2}=\left(\bar{A}+\bar{B} G_{z}^{*} T\right) \bar{S}_{1}+\bar{B} S_{2} \\ \bar{C} \bar{S}_{1}=0\end{array}\right.$

$\Leftrightarrow\left\{\begin{array}{l}S_{1} F=\left(A+B G_{z}^{*}\right) S_{1}+B S_{2}+\Gamma \theta \\ C S_{1}=Q \theta\end{array}\right.$
These Matrix Matching Equations can be uniquely solved for $\left(S_{1}, S_{2}\right)$ when $\mathrm{CB}$ is nonsingular (which it is when CB>0).

New Adaptive Controller:

$$
\left\{\begin{array}{l}
u=G_{e} \hat{e}_{y}+G_{z} \hat{z}+G_{D} \varphi_{D} \\
\dot{\hat{z}}=L_{21} \hat{e}_{y}+L_{22} \hat{z}+L_{23} u
\end{array}\right.
$$

with

$$
\left\{\begin{array}{l}
\dot{G}_{e}=-\hat{e}_{y} \hat{e}_{y}^{T} \gamma_{e} ; \gamma_{e}>0 \\
\dot{G}_{D}=-\hat{e}_{y} \varphi_{D}^{T} \gamma_{D} ; \gamma_{D}>0 \\
\dot{G}_{z}=-\hat{e}_{y} \hat{z}^{T} \gamma_{z} ; \gamma_{z}>0
\end{array}\right.
$$

Use

$$
\begin{aligned}
\hat{e}_{y} & \equiv y-Q \theta H_{D} \hat{z} \Rightarrow \dot{\hat{z}} \\
& =L_{21} \hat{e}_{y}+L_{22} \hat{z}+L_{23} u \\
& =L_{21} y+(\underbrace{L_{22}-L_{21} Q \theta H_{D}}_{L_{22}}) \hat{z}+L_{23} u
\end{aligned}
$$

Then

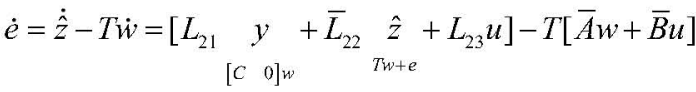

$$
\begin{aligned}
& =\left(L_{21}\left[\begin{array}{ll}
C & 0
\end{array}\right]+\bar{L}_{22} T-T \bar{A}\right) w+\bar{L}_{22} e=\left(L_{23}-T \bar{B}\right) u
\end{aligned}
$$

Choose $L_{23} \equiv T \bar{B} \quad$ where $T$ э $T \bar{A}-\bar{L}_{22} T=L_{21}\left[\begin{array}{ll}C & 0\end{array}\right]$ ( uniquely solvable when $\sigma(\bar{A}) \cap \sigma\left(\bar{L}_{22}\right)=\varphi$ ) with $\bar{L}_{22}$ chosen appropriately stable $\Rightarrow \dot{e}=\bar{L}_{22} e$

Also

$$
\begin{aligned}
& \Delta u \equiv u-u_{*}=u-\bar{S}_{2} z_{D} \\
& =u-\left(S_{2} z_{D}+G_{z}^{*} z_{*}\right) \\
& =u-S_{2} L_{D} \varphi_{D}-G_{z}^{*} z_{*} \\
& =G_{e}^{*} \hat{e}_{y}+\underbrace{\left(G_{D}^{*}-S_{2} L_{D}\right)}_{=0} \varphi_{D}+G_{z}^{*} \hat{z}-G_{z}^{*} z_{*} \\
& +\underbrace{\left[\begin{array}{ccc}
\Delta G_{e} & \Delta G_{D} & \Delta G_{z}
\end{array}\right]}_{\Delta G}\left[\begin{array}{c}
\hat{e}_{y} \\
\varphi_{D} \\
\hat{z}
\end{array}\right] \\
& =G_{e}^{*} \hat{e}_{y}+G_{z}^{*} \Delta \hat{z}+\Delta G \eta ; \Delta \hat{z} \equiv \hat{z}-z_{*} \\
& =G_{e}^{*}\left(\bar{C} \Delta w-Q \theta H_{D} e\right)+G_{z}^{*}(T \Delta w+e)+h \\
& =\left(G_{e}^{*} \bar{C}+G_{z}^{*} T\right) \Delta w+\left(G_{z}^{*}-Q \theta H_{D}\right) e+h
\end{aligned}
$$

so we can use adaptive control law (32) with gain adaption laws (33). 


\section{Proof that State Feedback does not Change the Minimum Phase of Transfer Function}

Assume a square transfer function $(\mathrm{M}=\mathrm{P})$.

The transmission zeros of $(A, B, C)$ are

$$
\begin{aligned}
& \lambda_{*} \ni \operatorname{rank}\left[\begin{array}{cc}
A-\lambda_{*} I & B \\
C & 0
\end{array}\right]<N+M \\
& \operatorname{rank}\left[\begin{array}{cc}
A-\lambda_{*} I & B \\
C & 0
\end{array}\right]=\operatorname{rank}\left(\begin{array}{cc}
A-\lambda_{*} I & B \\
C & 0
\end{array}\right] \underbrace{\left.\left[\begin{array}{cc}
I & 0 \\
G & I
\end{array}\right]\right)}_{\text {Non sin gular }} \\
& =\operatorname{rank}\left[\begin{array}{rr}
A+B G-\lambda_{*} I & B \\
C & 0
\end{array}\right] \\
& \Rightarrow \text { transmission zeros of }(A+B G, B, C) \\
& \text { are the same as those of }(A, B, C) \#
\end{aligned}
$$

\section{IMPLEMENTATION OF ADTC THEORY WITH PARTIAL} STATE ESTIMATION AND STATE FEEDBACK

\section{A. Simulation Setup}

We implemented the ADTC with partial state feedback to the NREL's 5 MW offshore wind turbine model [7]. This is three bladed horizontal axis upwind wind turbine with 63 meter of rotor radius and $5 \mathrm{MW}$ of rated power. The cut-in, rated and cut-out wind speed are $3 \mathrm{~m} / \mathrm{s}, 11.4 \mathrm{~m} / \mathrm{s}$ and $25 \mathrm{~m} / \mathrm{s}$ respectively. Also, cut-in and rated rotor speeds are $6.9 \mathrm{~m} / \mathrm{s}$ and $12.1 \mathrm{~m} / \mathrm{s}$ respectively. It has rated Tip Speed of $80 \mathrm{~m} / \mathrm{s}$ with a Tip Speed Ratio of 7.55.

Depending upon which Degree Of Freedom (DOF) we need, it has eleven switches which can be switched on and off to add the complexity in the operation. When all DOF's are switched on the wind turbine model consists 31 states. The detailed description of this wind turbine can be found in [7].

To design the wind speed and partial state estimation of wind turbine, the turbine is linearized at constant wind speed of $8 \mathrm{~m} / \mathrm{s}$ with blade pitch held at 0 degree. During the linearization Drive Train and Generator DOF switch were turned on which gave four states with two states due to the Generator DOF and two states due to Drive Train DOF. The first state (generator azimuth position) was removed to get the three state model of wind turbine since the linearized model becomes unobservable when included all four states. This three state model is then augmented with wind disturbance model given by equation (7) and state estimator was designed using equation (9).

\section{B. Simulation Results}

The ADTC with state estimator and state feedback designed is then implemented and simulated in NREL's $5 \mathrm{MW}$ onshore wind turbine in MATLAB/Simulink platform. We also simulated existing fixed gain PID controller [7] to compare the performance of ADTC.
The value of adaptive gains used were $\gamma \mathrm{D}=15000$ and $\gamma e=0.0001$. We obtained these values of adaptive gain by trial and error.

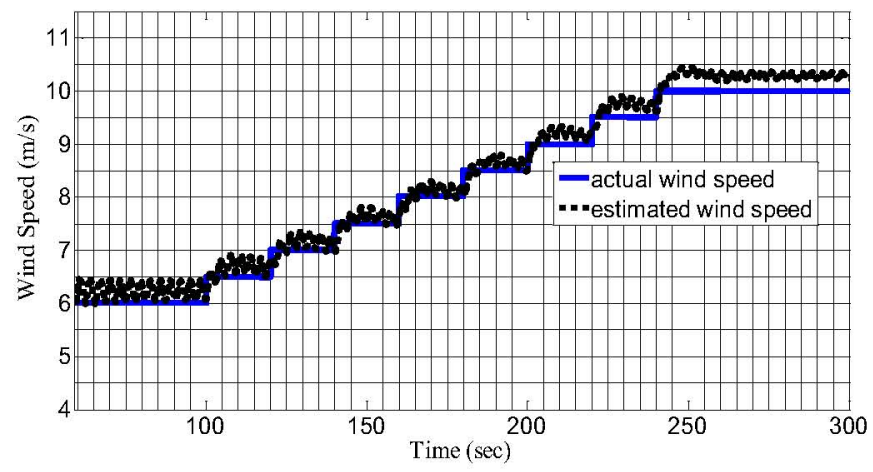

Figure 2. Actual vs estimated wind speed

Comparison of estimated wind speed with actual step wind profile is in figure 3 . The wind speed estimator combined with the partial state estimator has estimated the wind speed to a great extent.

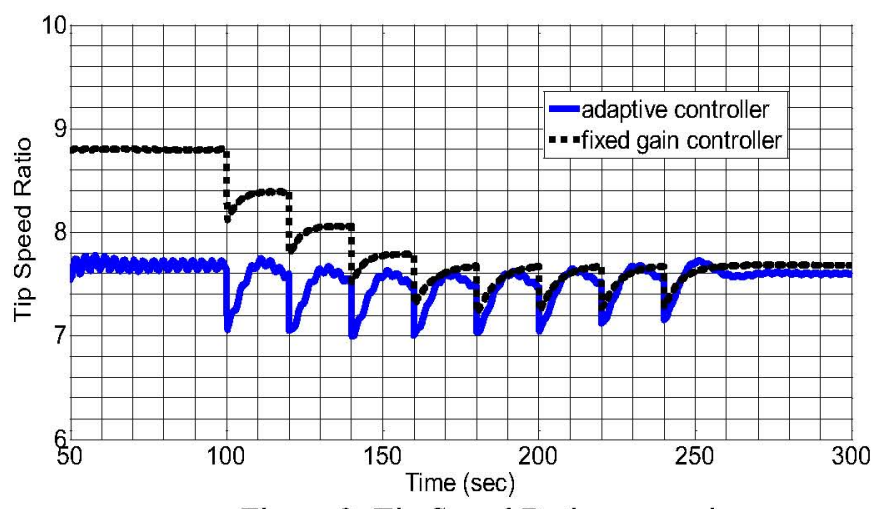

Figure 3. Tip Speed Ratio comparison

The TSR is compared between proposed ADTC and the existing fixed gain controller in figure 4 . In this simulation, the desired TSR is 7.55. The ADTC has better and consistent TSR tracking compared to the existing fixed gain controller.

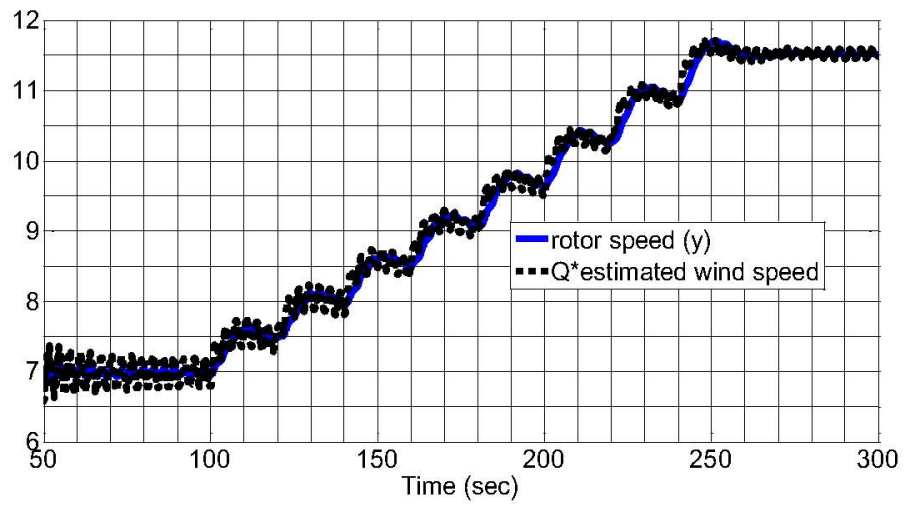

Figure 4. Comparison of rotor speed (yp) and $Q * \widehat{u} \mathrm{D}$ 
The components of estimated output tracking error given by equation (12) are shown in figure 5. In this simulation the value of tracking ratio $(\mathrm{Q})$ used was 1.12 . It can be seen that the adaptive controller with partial state feedback has done excellent job in disturbance tracking.

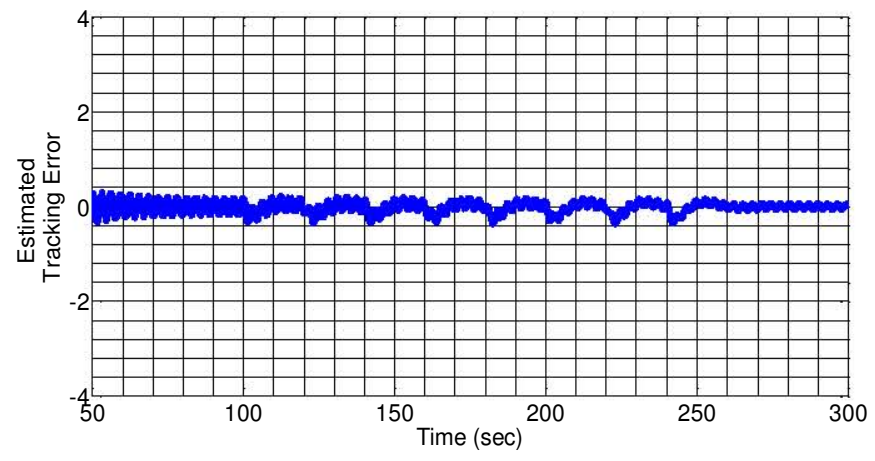

Figure 5. Estimated tracking error

The Estimated Tracking Error given by equation (12) is shown in figure 6 . The error is almost zero in steady state.

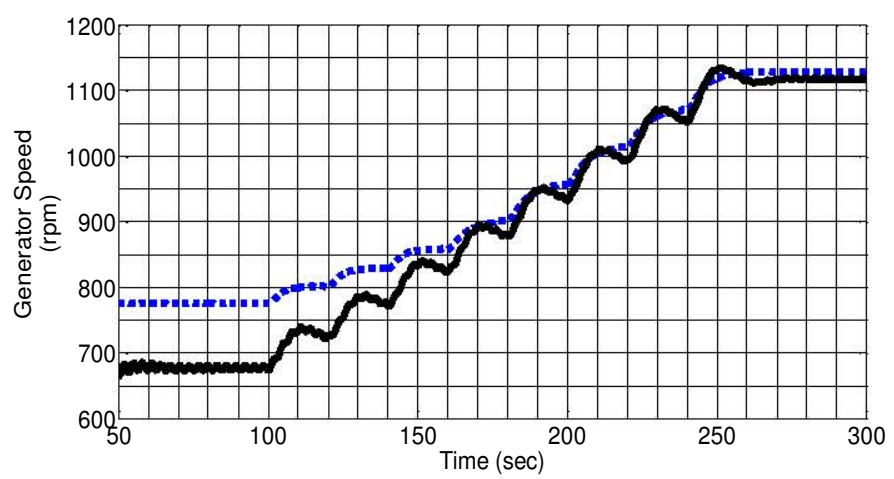

Figure 6. Generator speed comparison

The generator speed profile for the adaptive controller and the existing fixed controller is in figure 7. In lower wind speed region there is discrepancy between the generator speed, this is because the existing fixed gain controller the generator torque is directly the function of generator speed, given by $\mathrm{K} * \Omega^{2}$, where $\mathrm{K}$ is some constant, which depends on the desired TSR, and $\Omega$ is the generator speed. But, in adaptive disturbance tracking control, there is no direct relation between generator speed and torque, instead generator torque is manipulated in such a way that the generator speed track some percentage of wind speed.

The generator torque command input produced by both the adaptive controller and the fixed controller is shown in figure 8 . The adaptive controller has aggressive torque compared to the fixed gain controller. The reason behind this aggressiveness is tendency of adaptive controller to track the step wind speed. This aggressive behavior can be reduced to a great extent by using a low pass filter after estimated wind speed. This makes the wind speed smooth rather than step resulting the adaptive controller to follow the smooth wind speed.

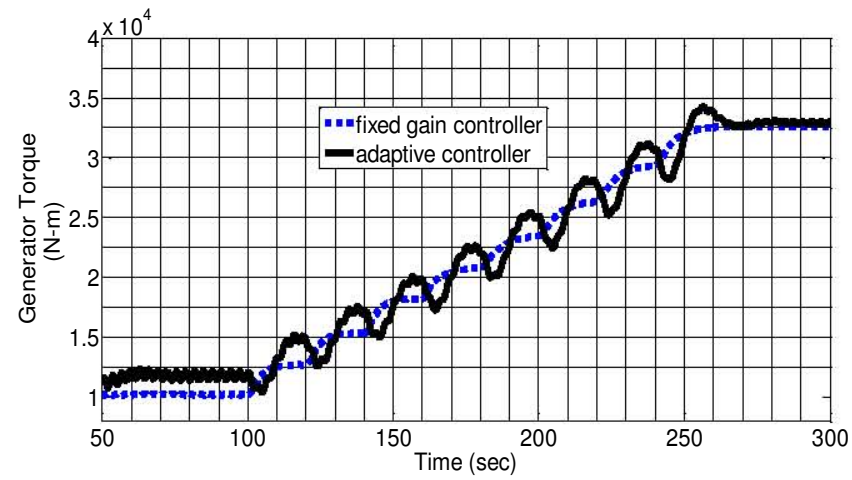

Figure 7. Generator Torque comparison

\section{CONCLUSION}

The theory of ADTC with partial state estimator and state feedback is proposed to track the TSR in Region II operation of large HAWTs, and its stability is also analyzed. The estimator/controller is designed based on proposed theory and simulated in $5 \mathrm{MW}$ onshore wind turbine. The main advantage of this theory is simplicity in design and omission of requirement of detailed model of the wind turbine. Also, parameters of wind turbines are poorly known which prevents design of controller using well established linear control theory but adaptive controller performs better in such situation.

\section{REFERENCES}

[1] Wilson, R.E., Lissaman, P., Applied Aerodynamics of Wind Power Machines, Corvallis, Oregon: Oregon state University, 1974.

[2] Wright, A. D., " Modern Control Design For Flexible Wind Turbines", Technical Report Submitted to National Renewable Energy Laboratory, Golden, CO, July 2004

[3] Johnson, C.D., Theory of disturbance-accommodating controllers, Control \& Dynamic Systems, Advances in Theory and Applications, Leondes, C. T. editor, Academic Press: New York, 1976; 12: 387-489

[4] Balas, M., Lee, Y., and Kendall, L., "Disturbance Tracking Control Theory with Application to Horizontal Axis Wind Turbines", Proceedings of ASME Wind Symposium, Reno, NV, AIAA-98-0032, Jan, 1998

[5] Balas, M., Thapa K., Frost, S.A., "Large Wind Turbine in Variable Speed Transition Operation: Adaptive Disturbance Tracking Control “, Proceedings of AIAA InfoTech 2012, Garden Grove, CA

[6] Thapa, K., Balas, M., and Frost, S.A., "Adaptive Disturbance Tracking Control with Wind Speed Reduced Order State Estimation for Region II Control of Large Wind Turbines", Accepted for ASME 2012 Conference on Smart Materials, Adaptive Structures and Intelligent Systems, September 1, 2012, Stone Mountain, Georgia

[7] J.M. Jonkman, M. L. Buhl, Jr. , 2007, "Development and Verification of a Fully Coupled Simulator for Offshore Wind Turbines", preprint and presented at the 45th AIAA Aerospace Sciences Meeting and Exhibits, Wind Energy Symposium, Reno, Nevada, Jan 